\title{
Maximum power point tracking in photovoltaic systems
}

\author{
Grażyna Frydrychowicz-Jastrzębska ${ }^{1, *}$ \\ ${ }^{1}$ Poznan University of Technology, Poland
}

\begin{abstract}
The subject of the analysis was the optimisation of interoperation between the photovoltaic battery (PV) and DC motor, which drives a fan, with respect to the maximum efficiency of conversion of the electric energy into mechanical energy. Based on the block diagram, a mathematical model of this circuit was developed to ensure the mutual matching between the Maximum Power Point (MPP) of the battery and the receiver operation point. A computer simulation of the battery characteristics was conducted taking into account the changing MPP location on the characteristic vs. changes in solar radiation and temperature. The issue was considered for the optimal motor excitation coefficient, both changing and averaged in time. The energy conversion efficiency was determined for selected PV modules, as well as time.
\end{abstract}

\section{Introduction}

The maximisation of the output power of the PV battery is obtained through the proper determination of the MPP location on its current-voltage characteristics. Interference with this position can take place by applying tracking algorithms, control systems or inverters provided with controllers [1-7]. The mutual matching of the MPP on the characteristic and the receiver operation point guarantees an increase in the efficiency of the system and thus the optimisation of its operation. This is confirmed by the test results [1-7]. The issue can be resolved for a specific interval as the MPP location on the characteristic changes, which is a consequence of changes of the characteristic itself, implied by differences in momentary irradiation levels $[4,5,6]$.

\section{Mathematical model of the circuit}

The mathematical model was developed on the basis of the theoretical model of the circuit which comprises a PV battery, a DC motor and a fan that constitutes a load. The battery operation was analysed and the currentvoltage characteristic as well as coordinates of the maximum power point (MPP) were determined [6]. There is a number of theoretical methods to resolve this issue, both based on the one- [3] and two-diode [6] PV battery diagram, starting from the second order NewtonRaphson iteration [6] through to the techniques of the maximum power point tracking, (Perturb i Observe, Neural Network) $[4,7]$. As has been demonstrated in $[3,5]$ both diagrams ensure the sufficient accuracy.

If the impact of the armature and the voltage drop under the brushes is ignored, the voltage equation of the separately excited DC motor looks as follows [5]:

$$
U=E+R_{t} I
$$

where: $U$-voltage, $E$-electromotive force (EMS) induced in the motor, $R_{t}$-armature resistance, $I$ - current.

EMS is the function of the engine $\omega$ speed and the excitation coefficient $c$. The proper selection of the coefficient ensures operation of the system nearby the MPP on the characteristic. Value $c$ is the function of design parameters of the motor and the excitation flux [5]:

$$
E_{0}=c \omega
$$

where: $\omega$ - angular velocity of the motor.

When limiting the losses to those resulting from the current flow through resistance $R_{t}$, the motor power is:

$$
P_{m}=U \cdot I-I^{2} R_{t}
$$

Fan torque $T$ is described as follows:

$$
T=B \cdot \omega^{2}
$$

where: $B$ - fan load constant.

The two-diode model was used to describe the currentvoltage characteristics and determine the MPP [5]. The curve which connected the set of these points within the considered interval was described as follows:

$$
U_{M P P}=k \cdot I_{M P P}
$$

where $U_{P M M}, I_{P M M}$-coordinates [5,6].MPP, $K$ and $\alpha$ approximation coefficients selected in such a way as to have equation (5) describe the consecutive MPP locations, $\alpha, k$ - coefficients selected for each battery. By equating the motor power derivative along the current coordinate to zero, the value of current which corresponds to the maximum of power was determined. Equations (6) and (7) were used to describe the values of current and voltage, following the changes in the motor power supply [5]:

$$
I=\frac{k \cdot I_{M P P}^{\alpha}}{R_{t}+k I_{M P P}^{\alpha-1}}
$$




$$
U=U_{M P P} \frac{2 \cdot R_{t}+k \cdot I_{M P P}^{\alpha-1}}{R_{t}+k \cdot I_{M P P}^{\alpha-1}}
$$

Coefficient $c$ was determined in such a manner as to enable power $\mathrm{P}_{\mathrm{m}}$ supplied to the motor correspond to the power value at MPP. As this value is different on each characteristic, this value is different [6], it is necessary to "control" the constant " $c$ " so as to obtain a close compliance of the motor characteristic wit the curve that describes the respective positions of the MPP. Value $c$ is presented in equation (8); the changes in the excitation are described by the battery parameters and load.

$$
c=B^{1 / 3} k^{2 / 3} I_{M P P}^{(2 \alpha-1) / 3}
$$

Based on the conducted analysis, the excitation coefficient as well as the optimal values of $U$ and $I$, which "follow" the changes in the motor power supply by the PV battery, were selected [5]. The determined values are both functions of constants $K$ and $\alpha$ as well as coordinates $U_{M P P}, I_{M P P}$ of MPP and rotor resistance $R_{t}$. Coefficient $c$ (8) allows the value of voltage and current under the conditions of optimal operation of the motor to be determined.

The solar radiation conditions change on a constant basis [6], therefore the optimal operation of the motor is not possible all day long without the tracker with tracking pursuit, and this, in turn, increases the investment costs. In practice, the averaged excitation value (weighted average) for the interval is determined. Such a selection of the coefficient usually ensures the required accuracy and optimisation of operation at simultaneous reduction in the number changes of its value. Complex systems are provided with more or less advanced control systems.

\section{Results of the computer simulation}

Based on the introduced relationships, a block diagram was prepared and the computer simulation was performed. Examples of the results of calculations of total energy for different design-operational variants are presented in Figure $1 \mathrm{~A}, \mathrm{~B}$ and in Figure $2 \mathrm{~A}, \mathrm{~B}$.

On the basis of the drawings, the influence of the procedure of selection the coefficient c (in particular the averaging interval, but also other conditions) on the efficiency of the system was found.

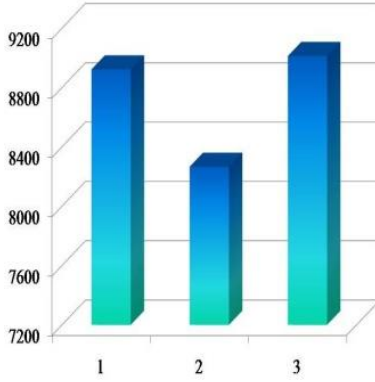

A

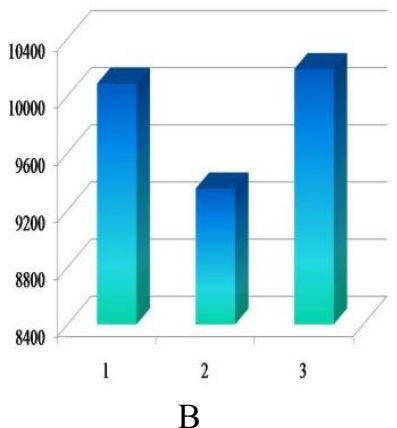

B
Fig.1. Comparative data regarding daily sums of energy in 3 cases: changing excitation coefficient (tracking) $\left(c_{v a r}\right)(\mathrm{a})$, averaged coefficient $c$ selected optimally for a specific interval (b), energy gained from PV conversion (c). Clarifications: time -1.06 , spatial orientation of the battery : angle of inclination to the ground $\beta=13^{\circ}$, azimuth angle $\gamma=2^{\circ}, T=25^{\circ} \mathrm{C}$, for case A: PV AEG PQ 40/50 batter, for case B : PV Siemens 50 battery.

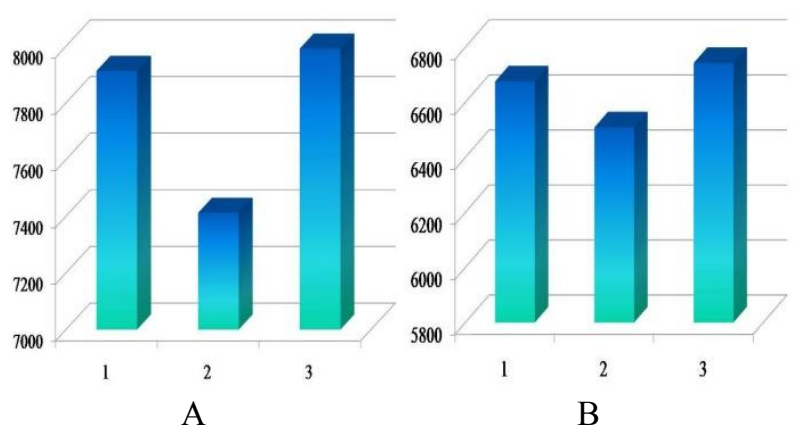

Fig. 2. Comparative data regarding daily sums of energy in 3 cases: changing excitation coefficient (tracking) $\left(c_{\text {var }}\right)$ (a), averaged coefficient $c$ selected optimally for a specific interval (b), energy gained from PV conversion (c). Clarifications: time 1.06, PV AEG PQ 40/50 battery, spatial orientation of the battery : angle of inclination to the ground $\beta=13^{\circ}$, azimuth angle $\gamma=2^{\circ}$, for case A: $T=25^{\circ} \mathrm{C}$, for case B: $T=50^{\circ} \mathrm{C}$.

\section{Summary and conclusions}

Based on analysis and simulations, it was concluded that:

- if the motor operation control system is used so that the change in the excitation could occur as a consequence of solar radiation, it is possible to achieve the efficiency even at the level of $99 \%$,

- the system efficiency reaches the highest values at hours with average insolation during a given day,

- the excitation coefficient values are lower for hours with a higher radiation power density,

- if the time of the circuit operation is limited, efficiency vs. $c_{\text {opt }}$ will reach a value which approximates the similar value during the matching of $c_{v a r}$,

- when the PV battery does not operate at MPP because of technological defects or shading, the maximum power supplied by it will be lower than the sum of maximum module powers,

- $\quad$ it is advantageous to track MPP by means of a micro-controller,

- benefits which follow from the control may be estimated only in the aspect of energy (3-7\%).

\section{References}

1. S. Bimenyimana, G. Asemota, L. Lingling, 2nd Intern. Conf. Power and Ren. Energy, 31-36, (2017)

2. A. Gupta, Y. Chauhan, Solar Energy, 136, 236-253, (2016)

3. M. A. Husain, A. Tariq, S. Hameed, M. Arif, A.Jain, Green Energy \& Environment,.1-13, (2016)

4. H. Islam, S. Mekhilef, N. Shah, T. Soon, M. Seyedmahmousian, B. Horan, A. Stojcevski, Energies 11, 365 1-24, (2018)

5. G. Jastrzębska, Ogniwa słoneczne, Wydawnictwa Komunikacji i Łączności, Warszawa, (2013)

6. G. Jastrzębska, Acad. Journ. El. Eng. 98,125135, (2019)

7. J. Ram, T. Babu, N. Rajasekar, N., Renew.and Sust. Energy Rev. 67, 826-847, (2017) 